\title{
Messages Prioritization in IEEE 802.15.4 based WSNs for Roadside Infrastructure
}

\author{
Imane Horiya Brahmi $\mp$, Soufiene Djahel ${ }^{\mp}$, Damien Magoni ${ }^{\ddagger}$ and John Murphy $\mp$ \\ $\mp$ UCD School of Computer Science and Informatics, Ireland \\ $\ddagger$ University of Bordeaux - LaBRI, France \\ \{horiya-imane.brahmi, soufiene.djahel, j.murphy\}@ucd.ie, magoni@labri.fr
}

\begin{abstract}
Wireless Sensor Networks (WSNs) have gained a wide popularity for real-time events monitoring and detection due to their high accuracy and ease of deployment. Therefore, they have become increasingly prevalent solutions in several application domains such as health-care, transportation, etc. Many studies in the literature have focused on optimizing the energy consumption of the wireless sensors in order to ensure their autonomous operation for longer periods. Some applications of WSNs, however, have strict delay requirements due to the nature of the information being transmitted. This delay should be kept as short as possible to ensure timely and efficient reaction of the system, such as a Traffic Management System (TMS) dealing with an incident on the road. In this context, we propose, in this work, two novel schemes to improve the transmission delay of prioritized messages in WSNs deployed in road networks to report regular traffic as well as event-driven (i.e. incidents) information. These schemes consist in an improvement of the backoff computation mechanism at the MAC layer of IEEE 802.15.4 standard protocol, in addition to an original dual mode operation mechanism, in order to speed up the transmission of event-driven messages, while keeping the transmission delay of periodic messages reasonably low. Simulation results show that our schemes succeed to reduce the transmission delay of eventdriven messages and achieve a very high packets delivery ratio. Keywords - Wireless Sensor Networks (WSNs), Road Monitoring, Smart Cities, IEEE 802.15.4, Backoff, MAC protocol, Intelligent Transportation System (ITS).
\end{abstract}

\section{INTRODUCTION}

Wireless Sensor Networks (WSNs) [12] involve a large number of sensor nodes that collaboratively collect, process and report different events. This technology is becoming increasingly popular for real-time events monitoring and detection, and for events sensing paradigm in general, spanning different application domains such as remote patients monitoring, underwater monitoring and transportation etc. In these applications, data collection is recognized as a fundamental process that plays a key role in determining the overall efficiency of the monitoring system. Nevertheless, collecting data without carefully considering aspects of time delay in some specific applications can result in serious issues in the monitoring process, ranging from lower system performance to human lives loss in case of emergency situations [11].

In most of WSNs based monitoring applications, the sensors sense continuously their environment and report the measured data periodically to the sink. However, there are a large number of other applications such as: gas or oil leakage detection, health monitoring to detect sudden change in patients vital signs, road traffic monitoring to detect random in-road incidents etc., where event-driven messages generation approach is more appropriate. These safety related applications that monitor and report emergency events occurrence require a very short transmission delay of the packets reporting such events, to ensure fast and efficient reaction from the system.
Among the above applications, road traffic monitoring, in the context of future smart cities, has recently attracted a lot of attention from the research community [1] due to the rapid growth of cities' population as well as their number of cars. This led to several ecological and economic problems especially in many industrialized countries. These problems require a substantial improvement of existing Traffic Management Systems (TMS) by, mainly, enhancing the efficiency of road monitoring equipment, particularly in big cities where traffic congestion presents a real challenge and the need for urban as well as highway traffic monitoring grows.

WSN is a promising solution to improve the efficiency and deployment flexibility of current road traffic monitoring equipment, such as induction loops, CCTV cameras etc. The application of WSNs technology in road traffic monitoring domain requires a high reliability for periodic messages transmission, and a very short delay for event-driven messages transmission, since these latter messages report critical information for drivers and passengers safety, hence the need of differentiated services between the different types of collected data.

In this paper, we consider the application of WSNs for real-time road events monitoring in smart transportation context, with focus on the design of alternative solutions to ensure fast transmission of event-driven messages (i.e. reporting an incident on the road), while maintaining the end to end transmission delay of other regular messages (i.e. reporting periodic measurement of some parameters) at an acceptable level for the overall system performance. To this end, we slightly modify the sensors MAC layer by designing a simple yet efficient dynamic Backoff Interval Adaptation (BIA) scheme for IEEE 802.15.4 standard protocol (un-slotted mode) [13]. This scheme is further enhanced by proposing an original dual mode operation mechanism to ultimately achieve the desired goals. Notice that we focus on the non-coordinated version of this protocol, also known as the peer-to-peer topology, because it avoids having a single point of failure (i.e., the coordinator in the star topology version) and multihop routing can be deployed easily on top of this topology. Notice that we use, throughout this paper, the terms event-driven messages and emergency messages interchangeably to refer to messages generated upon detection of an incident on the road.

The remainder of the paper is organized as follows. Section II presents the most relevant works in the literature. Next, we provide a detailed description of the proposed solution in section III. In section IV, we present and discuss the obtained simulation results of our designed solution under various simulation scenarios. Finally, we conclude the paper and discuss some future directions in section $\mathrm{V}$.

\section{RELATED WORK}

It is foreseen that WSNs will play a key role in improving road monitoring system efficiency. To this end, some works in the literature have illustrated the advantages of applying this technology in road networks. The sensors on the road can be static or mobile depending on which application they are used for. In this section, we 
discuss some works, and illustrate the advantages of applying WSNs technology in smart transportation.

The authors of [10] have proposed PEDAMACS which is a TDMA (Time Division Multiplexing Access) based MAC protocol in WSNs for road monitoring purposes. PEDAMACS applies a scheduling algorithm that guarantees the delivery of all packets to the sink at the end of each scheduling phase. The synchronization overhead is one of its major drawbacks, in addition to its restriction to one hop transmission only. Therefore, this makes it unsuitable for multihop and event-driven messages transmission due to the delay critical nature of such messages. Moreover, this protocol is not cost effective as its deployment at large scale requires a large number of costly sophisticated gateways. Hence, PEDAMACS is not an ideal solution for developing countries as well as cities lacking sufficient financial resources to deploy costly road monitoring equipment.

Most recently, Pascale et al. [3] have shown the benefits of WSNs technology for ITS applications, and how the wireless communication can increase the flexibility of road network monitoring. However the main focus of this work is the effective traffic information acquisition and processing rather than effective data collection techniques.

A data collection process becomes critical when wireless technologies are used for system monitoring purposes. In such a system, the data is usually reported periodically to the sink. However, the state of the network may change suddenly due to the occurrence of some incidents. In this case, event-driven messages need to be generated and transmitted as soon as the incident is detected, granting them the highest prioritized among other regular messages.

To manage this priority, numerous protocols using differentiated services have already been proposed in the literature. These protocols adapt the parameters of the standard IEEE802.15.4 according to the traffic type, for example, the authors of [4] proposed an enhancement of the Backoff algorithm by assigning different values to the three Backoff parameters (i.e., macMinBE, aMaxBE and CW) according to the type of traffic to be transmitted. Similarly, [5] adapts the CW (Contention Window) value according to the traffic type. However, these solutions are beacon enabled while our proposed scheme is designed to work in beacon-less mode.

In addition to MAC protocol enhancement, other solutions have focused on designing novel clustering algorithms tailored to WSNs to further improve data collection efficiency. The ultimate goal of such clustering mechanisms is to reduce the number of transmitted messages and thus increasing the network lifetime, and reducing the end to end delay. For further reading on this theme the reader may refer to: [7], [6], [8]. It is important to mention here that the clustering approach is out of scope of our work as we are focusing on improving event-driven messages transmission delay in IEEE802.15.4 protocol operating in beacon-less mode.

To conclude, WSNs represent a breakthrough technology for traffic monitoring that combines low-cost small vehicle detectors (powered by a battery and/or energy harvesting system) with scalable selfconfiguring wireless networking as described in [9] and [2].

\section{Proposed SOLUTION}

In this section, we present an overview of the system model followed by a description of our proposed solution which consists in a novel backoff adaptation scheme for achieving a better balance between event-driven and periodic messages transmission delay.

The architecture of our system consists of one gateway (i.e. WSNs sink) that collects traffic data reports from a large number of sensors deployed on the roadside as well as under the road pavement, as shown in Figure 1. In this architecture, we distinguish two different configurations of these sensors; the first set of sensors forms a linear topology where static nodes are arranged in parallel. In this configuration, we assume that these sensors are equipped with permanent energy supply, thus the problem of energy consumption is neglected. On the other hand, other sensors are deployed transversely, under the road pavement, one per lane across the road in order to

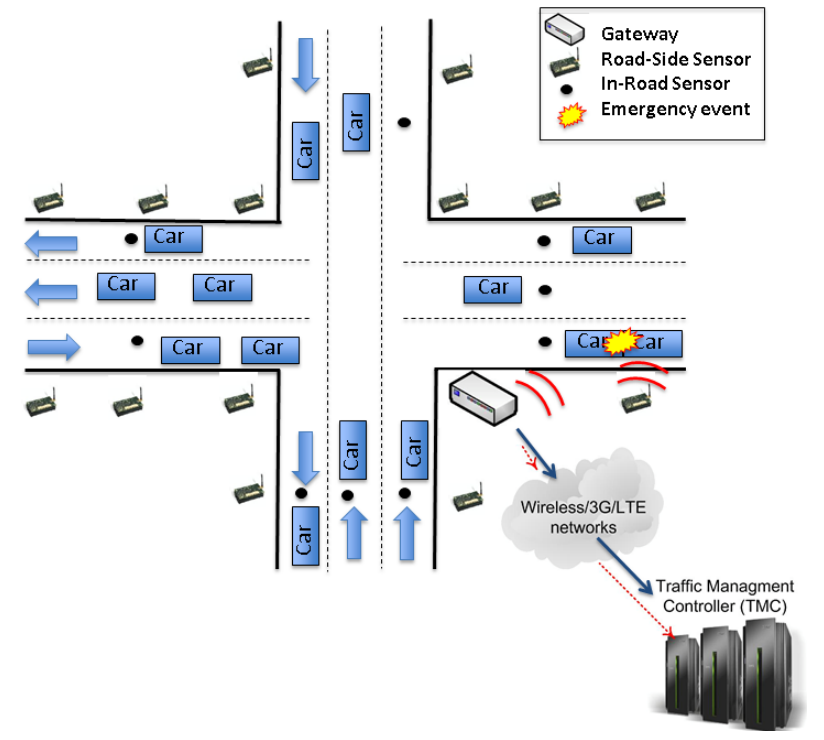

Figure 1: WSNs deployed in road networks for traffic flow monitoring and emergency events detection and reporting

collect and report data traffic information, such as the number of passing vehicles, their speed, road incidents and weather conditions (e.g., snow level). The WSN gateway is deployed in one corner of the road intersection, and has an important role in the synchronization of the other sensors. It is also used for managing the priority of the different packets received by using two types of queues with different priority levels: High and Low levels depending on the importance and the criticality of the messages. We connect the gateway to the Traffic Management Controller (TMC) via a set of wireless mesh routers constituting a Wireless Mesh Network (WMN) due to the offered flexibility of such network, and the low cost of installation and maintenance of the wireless technology compared to the existing wired infrastructure. Alternatively, we can also use cellular networks technology (e.g. 3G or LTE) to ensure the above connection.

The sensors in our architecture are deployed to measure and report two types of data which can be distinguished according to their urgency level. The messages carrying this data are classified into two main categories as described below:

- Class 1 (event-driven messages): these messages carry data regarding the detected incidents by the sensors, such as accidents (i.e. cars crash), a stalled car in the road or any other emergency event reported by the sensors mounted in the passing cars, such as emergency braking detection, etc. This type of data requires a minimum transmission delay from the detector sensor to the sink (i.e. a small backoff value for fast access to the medium) as well as reliable transmission. The occurrence of such events is random in time and space and the message carrying them have the highest priority at the gateway queue.

- Class 2 (periodic or regular messages): these messages are transmitted regularly and carry traffic and weather conditions related data. The weather conditions represent temperature, pressure and humidity etc. The data collected for traffic conditions are usually vehicles speed, traffic flow speed and volume, road occupancy level etc. These parameters are measured periodically and represent the lowest priority class (i.e. they are less critical for TMS efficiency).

The main objective of our work is to reduce the end to end transmission delay of the messages belonging to the Class 1 described above. To this end, we have designed a novel backoff adaptation scheme to meet the requirements of event-driven messages transmis- 
sion.

Since we have to transmit different types of data with different priority levels, the challenge in this case is to speed up the channel access for a sensor reporting a detected emergency event. To face this challenge, we propose to adapt the Backoff Interval (BI) to the class of the message to be transmitted as explained, in detail, in the following sub-sections.

\section{A. Backoff Interval Adaptation (BIA) scheme}

To achieve our objective, we divide the backoff interval into two parts: the first part of the interval $\left[0,2^{B E-1}\right]$ is dedicated to eventdriven messages (Class 1 ), while the second half $\left[2^{B E-1}, 2^{B E}\right]$ is reserved for the periodic or regular messages (Class 2), as shown in Figure 2. This scheme grants smaller backoff values for event-driven messages over the other types of messages, hence ensuring a lower end to end delay. The backoff selection mechanism follows a uniform distribution, in both classes 1 and 2, to give an equal transmission probability to all the sensors aiming to transmit the detected/received emergency events and minimize the collision probability.

Assuming $X$ a random variable that represents the backoff delay and its value varies within the interval $\left[0,2^{B E}\right]$, hence $X$ can be defined as follows:

$$
\begin{aligned}
& X \mid C=1 \sim U\left(\left[a_{1}, b_{1}\right]\right) \\
& X \mid C=2 \sim U\left(\left[a_{2}, b_{2}\right]\right)
\end{aligned}
$$

Such that:

- $a_{1}=0$

- $b_{1}=a_{2}=2^{B E-1}$

- $b_{2}=2^{B E}$

Where $C$ refers to the class of the message to be transmitted over the wireless channel.

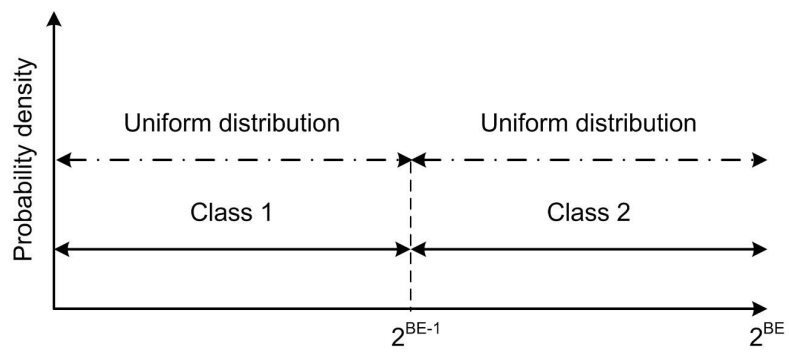

Figure 2: The backoff distribution used in the BIA.

\section{B. Mode switching based BIA}

We improve the above BIA scheme by introducing two modes of operation in which the sensors use different BI's to transmit both event-driven and periodic messages. The idea behind this is to avoid penalizing the periodic messages when there are no event-driven message being sent or forwarded by the sensor or its immediate neighbors. To this end, we define two modes of operation: the Urgent Mode and the Normal Mode as illustrated in the Figure 3. As defined in section III-A, an event-driven message backoff is chosen from $\left[0,2^{B E-1}\right]$ while a periodic message backoff is chosen from the interval $\left[2^{B E-1}, 2^{B E}\right]$. This mode is referred to as the Urgent Mode used only in case of reception or generation of an event-driven message. We define a second mode, the Normal Mode, which is set in absence of event-driven messages. We choose to pick our backoff for periodic messages in this mode from the whole interval $B I$. The random variable $X$ of the class 2 in normal mode is defined as follows:

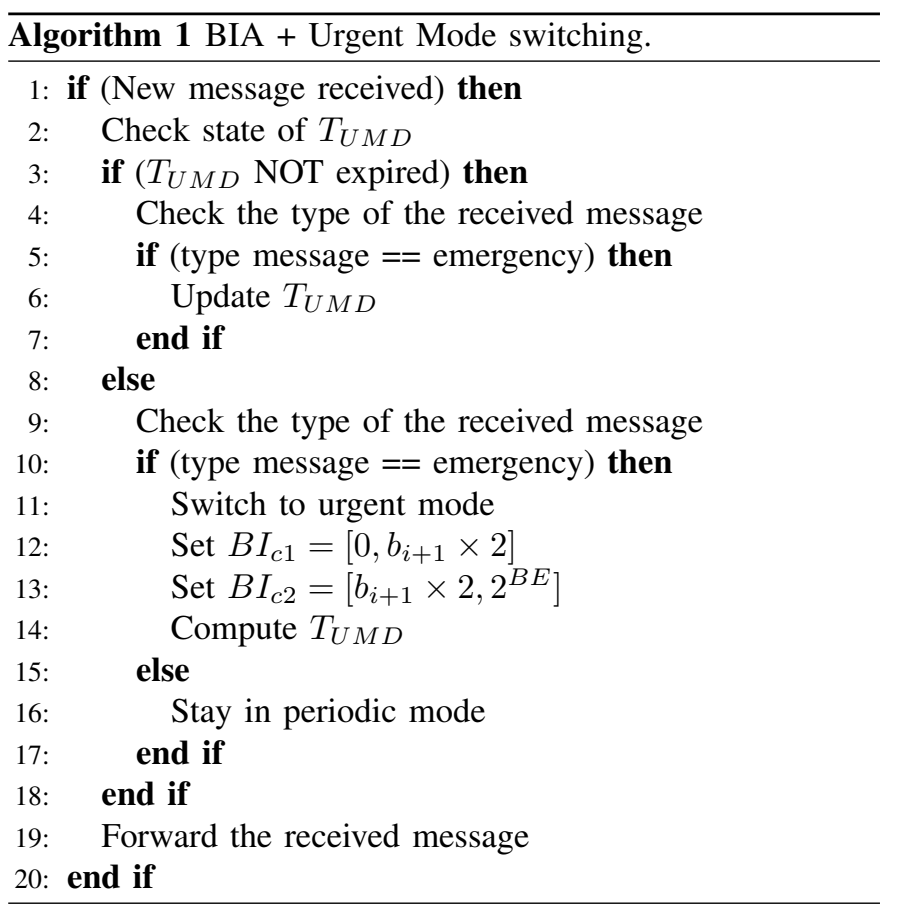

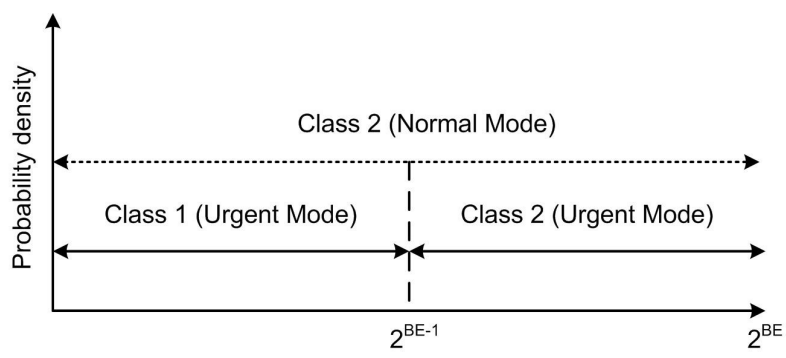

Figure 3: The backoff distribution used in mode switching based BIA.

1) Urgent Mode operation: the main challenge in using two different modes of operation is to define how long a sensor node should stay in urgent mode after receiving an event-driven message. To this end, we need to design an accurate approach to dynamically set the Urgent Mode Duration (UMD) value. In this context, whenever a sensor node receives an event-driven message it switches its transmission mode from normal to the urgent mode, then it calculates the UMD value and remains in this mode until the expiration of this UMD. The Algorithm 1 illustrates the different cases where a sensor node switches between the two modes, as described in the following steps:

1) Each time an event-driven message is received, the node switches to the urgent mode, and computes the value of its $T_{U M D}$.

2) If a new message is received, we check the state of $T_{U M D}$.

3) If $T_{U M D}$ has expired, and the received message is an eventdriven message, then the message will be sent under the urgent mode; if it is a periodic message, the backoff will use all the $B I$.

4) If $T_{U M D}$ has not expired yet, and the received message is of emergency type, $T_{U M D}$ will be updated. If it is a periodic 
message, it will then be sent under the urgent mode until $T_{U M D}$ expires, then the node will switch back to the periodic mode.

The major benefits of adopting these two modes by the sensors can be summarized in following:

1) Preventing the penalization of periodic messages when there is no event-driven message received; the periodic messages can draw their backoff from the full BI as defined in the periodic mode, instead of picking a backoff from $\left[2^{B E-1}, 2^{B E}\right]$ as defined in BIA.

2) Another advantage of this scheme is that when the intermediate node receives an event-driven message, it has to switch to urgent mode. Let consider the topology with three nodes $A_{1}, A_{2}$ and $A_{3}$ as shown in Figure 4. The nodes $A_{1}$ and $A_{2}$ are in the same transmission range, while $A_{3}$ is two hops away from $A_{1}$. If $A_{2}$ receives an event-driven message from $A_{1}$, then it will forward it to the next hop $A_{3}$ and choose the backoff from the interval $\left[0,2^{B E-1}\right]$. At the same time if $A_{1}\left(A_{3}\right)$ has to send a periodic message, then it will pick its backoff from the interval $\left[0,2^{B E}\right]$. In this case, the backoff of $A_{1}\left(A_{3}\right)$ can be smaller than the backoff of $A_{2}$ and the periodic message will be sent before the urgent message. Therefore, this doesn't meet our objective which is: "send (forward) the urgent message with minimum delay". However, if the node $A_{1}\left(A_{3}\right)$ sets the appropriate value of UMD and waits till its expiration, the urgent message will be sent and forwarded by the hop node within a short delay.

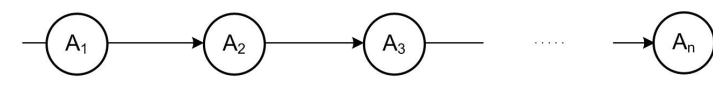

$A_{1}$

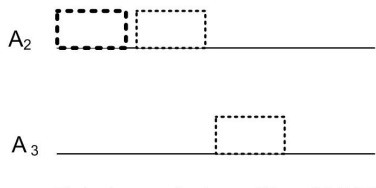

Data transmission without UMD

Emergency Message Sent

Periodic Message Sent

UMD
$A_{1}$
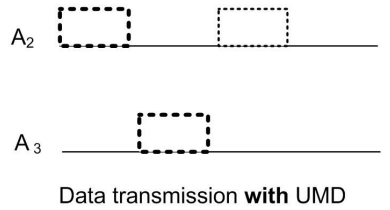

Emergency Message Recieved

Periodic Message Recieved
Figure 4: Example illustrating the benefits of using the Urgent Mode Duration (UMD).

The value of UMD is based on the observations of the previous monitoring periods (i.e., time windows) as shown in Figure 6. Let's divide the sensor's lifetime into periods $\left\{T_{i}\right\}_{i \geq 0}$. The periodic messages are sent periodically in each period, while the event-driven messages are generated upon detection of an emergency event on the road. Each sensor measures information regarding road traffic state, weather conditions, etc. and sends these readings periodically (i.e. at the end of each time period $\left\{T_{i}\right\}_{i \geq 0}$ ) towards the gateway. Upon reception (generation) of an event-driven message, the $U M D_{i}$ value is updated according to the inter arrival time of the event-driven messages during $T_{i-1}$.

2) UMD calculation: we define the UMD value by taking a statistical characteristic (e.g., average, $n$-th percentile, etc) of the distribution of the intervals separating two consecutive event-driven messages. Our mechanism obtains this distribution by storing the frequency of the inter-arrival time intervals of the event-driven messages collected during the previous time window of the sensor. These intervals can be aggregated for coping with memory limitations. We thus define the Emergency Events Inter-arrival Duration (EEID) as the time separating the occurrence of the $m$-th and $m-1$-th emergency events:

$$
E E I D_{m}=E E_{m}-E E_{m-1}
$$

Once the EEIDs are collected for a given time window, we calculate the Cumulative Distribution Function (CDF) of those values (an example is shown in Figure 5). Then our mechanism sets the UMD value by taking the 75-th percentile value of the CDF of the EEID. This 75-th percentile threshold has been used in all the simulation results of Section IV. In the example of Figure 5, the UMD would be set to approximately 18,5 seconds.

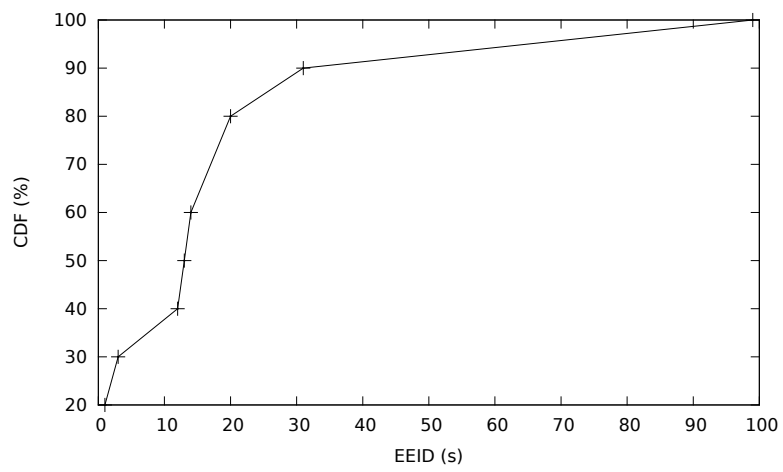

Figure 5: Example of a Cumulative Distribution Function of the EEIDs

\section{PERformance EVAluation}

In this section, we present the chosen simulation topology, parameters setting and discuss the obtained results. The simulations are performed using the $n s 2.35$ network simulator [14] under which the performance of the proposed algorithms has been evaluated. To carry out the simulations, we have modified the existing implementation of the IEEE 802.15.4 protocol in $n s-2$ by adding new functions to the file $802.15 .4 \mathrm{csmaca}$.cc. The primary metrics to be evaluated in these simulations are the End-to-End (E2E) transmission delay and the Packet Delivery Ratio (PDR) of both event-driven and periodic messages.

Table I: Summary of simulation parameters

\begin{tabular}{ll}
\hline Parameters & Value \\
\hline Transmission range & $10 \mathrm{~m}$ \\
Traffic type & CBR \\
Routing Protocol & AODV \\
Propagation model & FreeSpace \\
Total number of sensors & $25,50,75,100$ \\
Size of event-driven messages & 64 Bytes \\
Size of periodic messages & $63 \mathrm{Bytes}$ \\
Data rate & $40 \mathrm{kbps}$ \\
Transmission frequency of periodic msgs & $15 \mathrm{~s}, 20 \mathrm{~s}, 30 \mathrm{~s}, 60 \mathrm{~s}, 90 \mathrm{~s}, 120 \mathrm{~s}$ \\
Proportion of event-driven msgs & $4 \%$ \\
Simulation time & $600 \mathrm{~seconds}$ \\
No. of simulation runs & 10 \\
\hline
\end{tabular}

\section{A. Simulation setup}

1) WSN topology: We have applied our proposal on a freeway topology, where we have set up a single gateway and deployed several sensors transversally and in parallel to the road. An example 


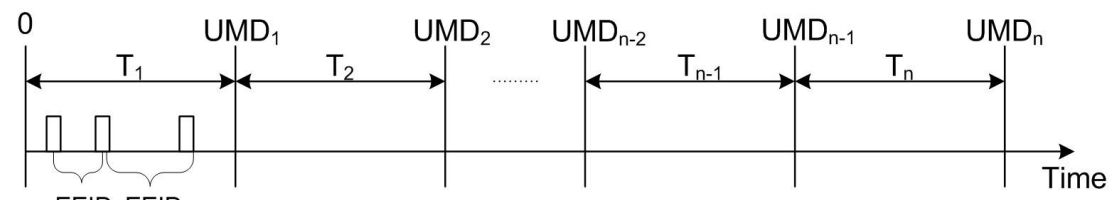

$\mathrm{EEID}_{1} \mathrm{EEID}_{2}$

EEID ${ }_{\mathrm{i}}$ : Time interval separating the detection (reception) of two consecutive emergency events (notification)

\section{Emergency events}

Figure 6: The variation of UMD value based on the EEID during WSN lifetime.

of this configuration is shown in Figure 7. The distance between the road-side sensors is set to 10 meters while the in-road sensors are spaced by 3.33 meters. Due to the short distance between the nodes we have used the FreeSpace propagation model and set the carrier sense and receiver threshold distances to 4 meters. The chosen value for the UMD is equal to the 75-th percentile of the measured EEID. In this configuration, all the sensors, except the gateway, transmit or forward two categories of messages: periodic and event-driven packets. The packets of the first category are generated periodically with an interval varying from 15 to 120 seconds following a CBR traffic pattern, while the event-driven packets are generated following a Poisson distribution. Notice that the emergency events occur randomly during the simulation time and their proportion varies between $4 \%, 8 \%$ and $16 \%$ of the total number of generated periodic messages. The packet size of the periodic and event-driven messages is fixed to 63 bytes and 64 bytes respectively, and both of them are transmitted at 40 kbps. The other simulation parameters are summarized in the Table I.

2) Modeling emergency events inter-arrival time: we assume that the emergency events occurrence times follow a Poisson distribution. The Poisson distribution is the most common choice for modeling discrete events which occur randomly. Thus the time duration between two emergency events is given by an exponential distribution as shown below. Consider $N$ a discrete random variable which models the number of emergency events during a time window $T$. We denote by $n \geq 0$ the different possible realizations of $N$. The random variable $N$ follows a Poisson distribution with the parameter $\lambda$ such that:

$$
\mathbb{P}[N=n]=\exp (-\lambda) \frac{\lambda^{n}}{n !}
$$

where,

- $\mathbb{P}[N=n]$ : probability that $n$ emergency events occur.

- $N$ : random variable representing the occurrence of emergency events during a given time window.

- $\lambda$ : Poisson parameter, the expected emergency events during each time window.

To calculate $\lambda$, we assume that we have $k$ observations $n_{1}, \ldots, n_{k}$ representing different numbers of emergency events which have occurred during the time window $T_{1}, \ldots, T_{k}$. Then, $\lambda$ is estimated with the sample mean:

$$
\lambda=\frac{1}{k T} \sum_{i=1}^{k} n_{i}
$$

Let us consider the emergency event times as a random variable $X_{m} \quad(m \geq 0)$ that represents the occurrence time of the $m$ th emergency event. We define the Emergency Events Inter-arrival Time $\left(E E I D_{m}=X_{m}-X_{m-1}\right)$ as the time interval separating the occurrence of the $m$-th and $m-1$-th emergency events. It is then acknowledged that the random variable $E E I D_{m}$ follows an exponential distribution with parameter $\lambda$. Therefore we have:

$$
\mathbb{E}\left[E E I D_{m}\right]=\frac{1}{\lambda}
$$

In our simulations, we have thus generated emergency events whose inter-arrival time intervals do follow an exponential distribution.

\section{B. Analysis of the simulation results}

In our simulation, we compare the two proposed algorithms, BIA and (BIA+UM), with the standard IEEE802.15.4 algorithm. Notice here that UM means that the urgent mode switching mechanism is enabled. The histograms plotted in Figure 8 compare the average E2E delay of all the messages (periodic and event-driven) under various values of the transmission interval of periodic packets (i.e. Inter Packets Transmission Interval (IPTI)). In this scenario, we set the event-driven messages' ratio to a fixed value equals to 4 $\%$ and the network size to 50 nodes; while we vary the IPTI of periodic messages from 15 to 120 seconds. We run our simulations with the standard algorithm and then with BIA and (BIA+UM) algorithms. The results depicted in Figure 8(b) reveal that our two algorithms achieve a shorter average E2E delay of event-driven messages compared to the standard algorithm. This is due to the higher priority granted to event-driven messages in our algorithms through the use of a smaller $B I$. On the other hand, the results in 8(a) show that the E2E delay of BIA can be equal or slightly higher than the standard in some cases, for example when the IPTI is equal to 20, 30 and 120 seconds. However, (BIA+UM) archives always shorter delay than both the standard and BIA, which clearly highlights the efficiency of the proposed urgent mode switching mechanism. This efficiency of (BIA+UM) decreases with the increase if IPTI as the larger value of IPTI leads to less traffic load on the network, which reduces the collision probability and thus speeds up the transmission of both categories of messages. On the other hand, a small value of IPTI leads to a considerable increase of the traffic load, which consequently increases the E2E delay for both categories of messages.

Notice also that, as opposed to our two algorithms, the E2E delay of event-driven messages sent using the standard algorithm is larger than the E2E delay of periodic messages sent by the same algorithm. The corresponding PDR of this scenario, as shown in Figure9, shows that the significant improvement brought by (BIA+UM) scheme doesn't reduce the PDR value as it similar to that achieved by the standard algorithm in most cases. It is worth to mention that the lack of a priority mechanism tailored for event-driven messages in the standard algorithm leads to an increase of their transmission delay compared to the periodic messages. The results in Figure 8 highlight this fact since the achieved delays of event-driven messages are higher than those of the periodic messages, especially for the IPTI values equal to $15,20,30$ and 60 seconds.

Figures 10(a) and 10(b), show the impact of the network size on the average E2E transmission delay for periodic and event-driven 


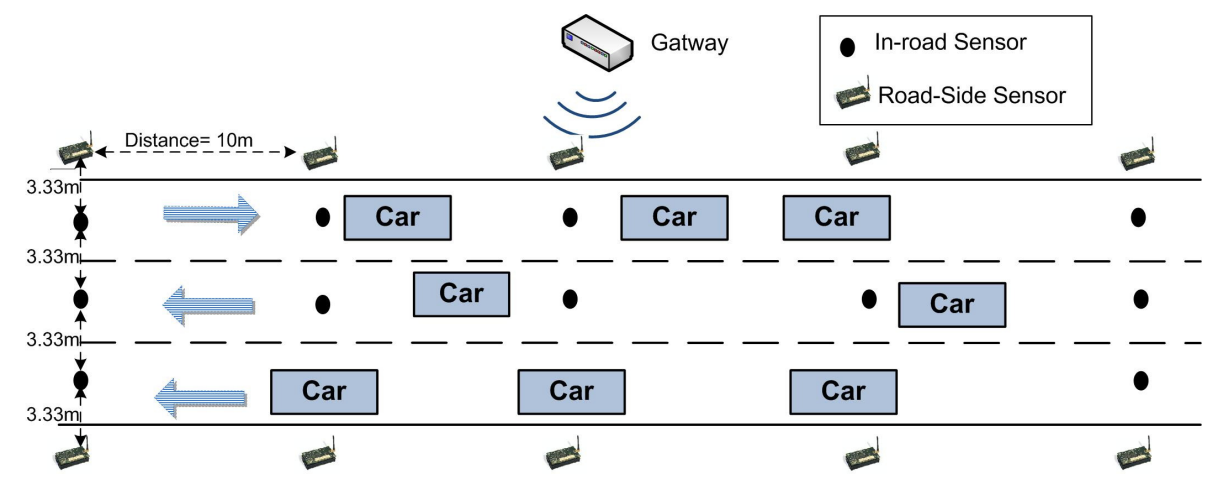

Figure 7: The road network topology used in our simulation

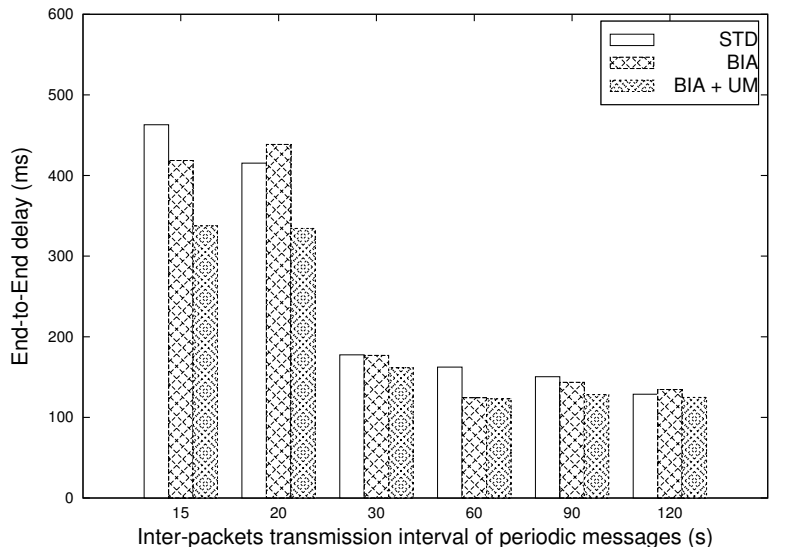

(a) Periodic messages

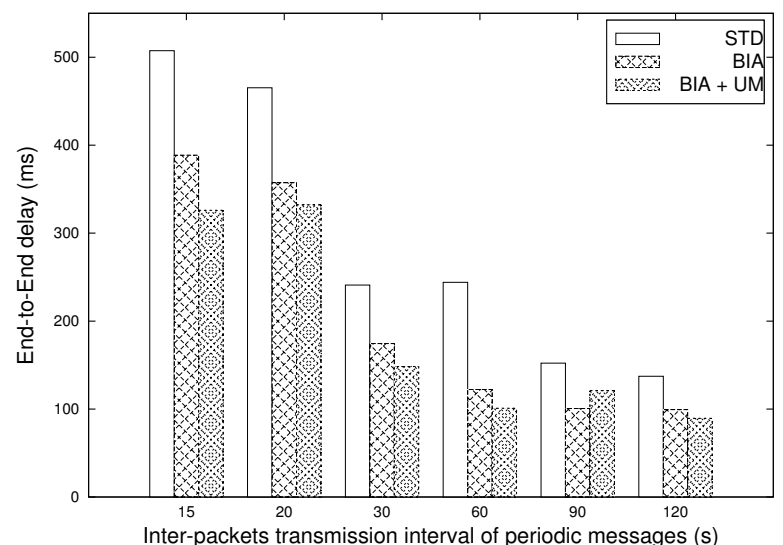

(b) Event-driven messages

Figure 8: Impact of inter-packets transmission interval on Average End-to-End delay: Proportion of event driven messages $=4 \%$, Network size $=50$

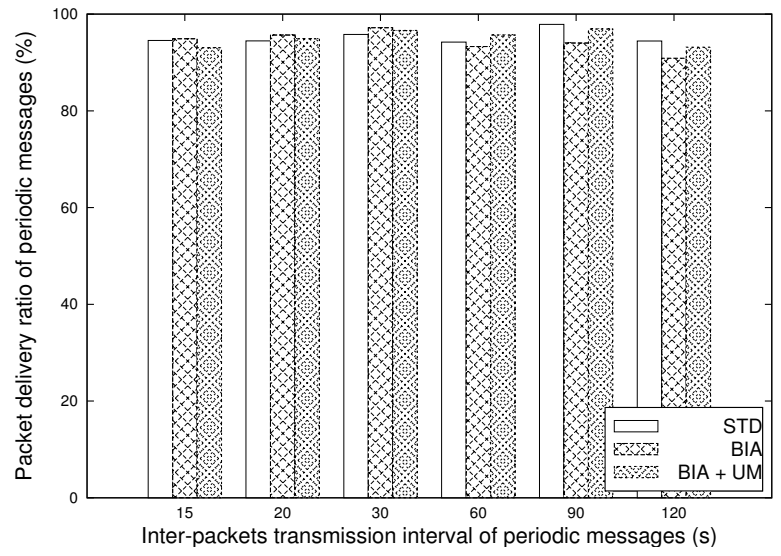

(a) Periodic messages

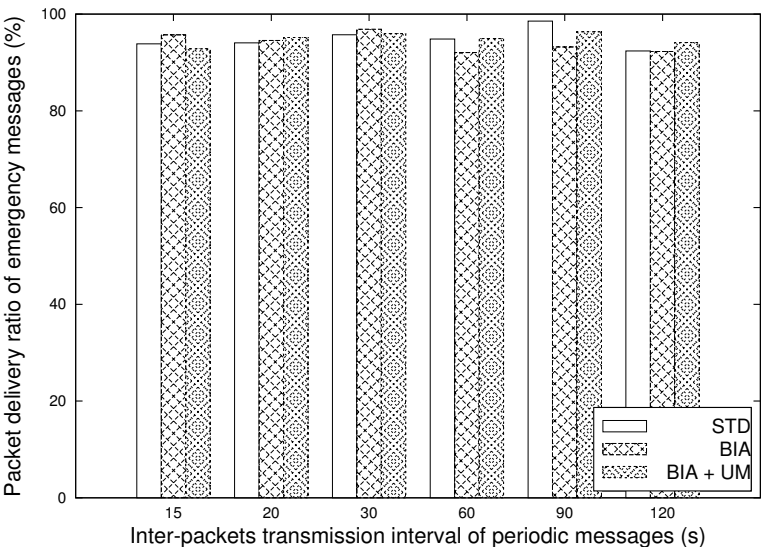

(b) Event-driven messages

Figure 9: Impact of inter-packets transmission interval on Packets Delivery Ratio: Proportion of event-driven messages $=4 \%$, Network size $=50$ 
messages, respectively. With the increase of the network size, the number of hops separating each node with the sink increases. The results plotted in these figures show that the E2E delay increases with the increase of the network size. Despite this increase, our algorithms deliver the event-driven messages faster than the standard (see Figure 10(b)). A lower delay is also observed for periodic messages using the (BIA+UM) algorithm (see Figure10(a)) compared to that achieved by the BIA algorithm.

Notice that in some cases, a slight increase in the delay of periodic messages can be observed, for example when the network size is equal to 75 and 100 nodes (see Figure 10(a)). As the periodic messages are usually carrying information that do not have strict delay requirements, prioritizing the event-driven messages can lead to the increase of the E2E delay of these periodic messages. However, the E2E delay of event-driven messages using the standard algorithm, for the same values (i.e. 75 and 100 nodes), is higher than the BIA and (BIA+UM) algorithms. Since the objective of our work is to achieve a lower E2E delay for event-driven messages delivery, the slight increase of the E2E delay of the periodic messages is a small price to pay.

So far, we have discussed the performance improvements of our proposed algorithms compared to the standard one. We can conclude that our proposed (BIA+UM) algorithm achieves a significant improvement over the standard algorithm in terms of E2E delay of event-driven messages, and keeps almost the same E2E delay for periodic messages as the one achieved by the standard algorithm. Moreover, it ensures a similar PDR to the standard algorithm.

\section{CONCLUSION AND FUTURE WORK}

In this paper, we have proposed two algorithms to improve the latency of event-driven (emergency) messages in WSNs-based road traffic monitoring. More precisely, we managed to reduce the transmission delay of critical events while keeping their delivery ratio as high as possible. The first solution consists in adapting the Backoff Interval to assign small backoff time to the messages reporting critical events in order to favor their transmission over other periodic messages. The second algorithm is an enhancement of the previous one, and consists in designing two transmission modes: urgent and normal. The urgent mode is used only in case of generation or reception of event-driven messages, while the normal mode is set in absence of these messages. We have also introduced the Urgent Mode Duration UMD which is the required time for a sensor node to stay in the urgent mode before it switches back to the normal mode. This technique allows us to avoid penalizing the periodic messages when there is no emergency activity going on. Our algorithms have been implemented in $n s-2$ and the obtained results have proven their efficiency under different values of input parameters such as the transmission interval of periodic messages and the size of the network. As for future work, we plan to investigate data aggregation techniques based on spatial correlation in WSNs in order to reduce the traffic load in the network, and thus increase the delivery ratio when the network becomes saturated.

\section{ACKNOWLEDGMENTS}

This work was supported, in part, by Science Foundation Ireland grant 10/CE/I1855 to Lero - the Irish Software Engineering Research Centre (www.lero.ie). This work was also supported by the Telecommunications Graduate Initiative (TGI) which is funded by the Higher Education Authority under the Programme for Research in ThirdLevel Institutions (PRTLI) Cycle 5 and co-funded under the European Regional Development Fund (ERDF).

\section{REFERENCES}

[1] P. Mohan, V. N. Padmanabhan and R. Ramjee, "Nericell: rich monitoring of road and traffic conditions using mobile smartphones," in Proceedings of the 6th ACM conference on embedded network sensor systems, SenSy' 08, November 04-07, Raleigh, NC, USA, 2013.
[2] S. Cheung and P. Varaiya, "Traffic surveillance by wireless sensor networks: Final report," Technical report UCB-ITS-PRR-2007-4, University of California, Berkeley, USA, Tech. Rep., 2007.

[3] A. Pascale, M. Nicoli, F. Deflorio, B. Dalla Chiara, and U. Spagnolini, "Wireless sensor networks for traffic management and road safety," Intelligent Transport Systems, IET, vol. 6, no. 1, pp. 67-77, 2012.

[4] K. Anis, M. ALVES, B. NEFZI and Ye-Q, SONG, "Improving the IEEE 802.15. 4 slotted CSMA/CA MAC for time-critical events in wireless sensor networks." Proceedings of the Workshop of Real-Time Networks, RTN'06, May 15-22, CSEM Switzerland, 2006.

[5] E. D. Ngangue Ndih, N. Khaled and G. De Micheli, "An Analytical Model for the Contention Access Period of the Slotted IEEE 802.15.4 with Service Differentiation", in IEEE International Conference on Communication, ICC'09, June 14-18, Dresden, Germany, 2009.

[6] I. S. AlShawi, L. Yan, W. Pan and B. Luo "Fuzzy chessboard clustering and artificial bee colony routing method for energy-efficient heterogeneous wireless sensor networks." International Journal of Communication Systems, 2013.

[7] N. D. Pham, N. D. Pham, T Duc Le2, K. Park and H. Choo, "SCCS: Spatiotemporal clustering and compressing schemes for efficient data collection applications in WSNs" in International Journal of Communication Systems, vol. 23, no. 11, pp. 1311-1333, 2010.

[8] M. Khiati, and D. Djenouri. "BOD-LEACH: broadcasting over dutycycled radio using LEACH clustering for delay/power efficient dissimilation in wireless sensor networks." International Journal of Communication Systems, 2013.

[9] M. Tubaishat, P. Zhuang, Q. Qi, and Y. Shang, "Wireless sensor networks in intelligent transportation systems," Wireless communications and mobile computing, vol. 9, no. 3, pp. 287-302, 2009.

[10] S. C. Ergen and P. Varaiya, "Pedamacs: Power efficient and delay aware medium access protocol for sensor networks," Mobile Computing, IEEE Transactions on, vol. 5, no. 7, pp. 920-930, 2006.

[11] W. Feng, L. Jiangchuan. "Networked wireless sensor data collection: Issues, challenges, and approaches" Communications Surveys \& Tutorials, IEEE, vol. 13, no. 4, pp. 673-687, 2011.

[12] Akyildiz, Ian F., et al. "Wireless sensor networks: a survey." Computer networks, vol. 38, no. 4, pp. 393-422, 2002.

[13] I. L. S. Committee, "Std.802.15.4: standard for local and metropolitan area network, part 15.4: low-rate wireless personal area networks (lrwpans)," IEEE Standards Association, Tech. Rep., 2011.

[14] The Network Simulator ns-2 (http://www.isi.edu/nsnam/ns/) 


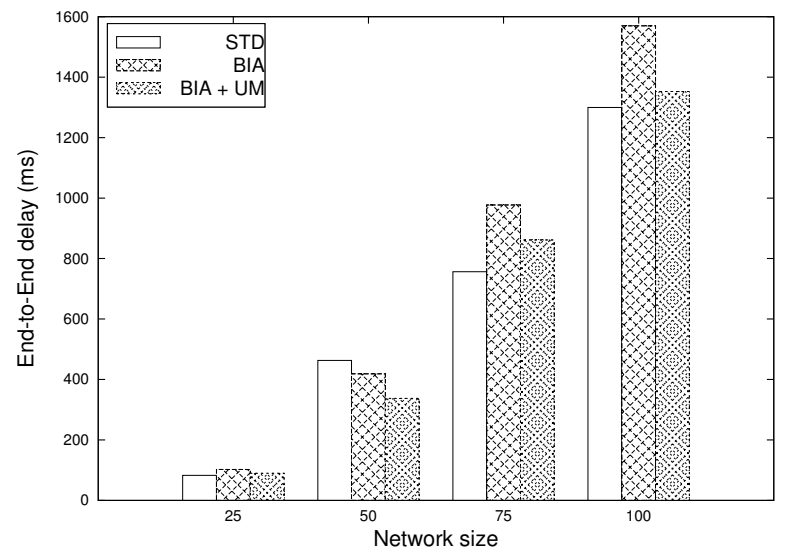

(a) Periodic messages

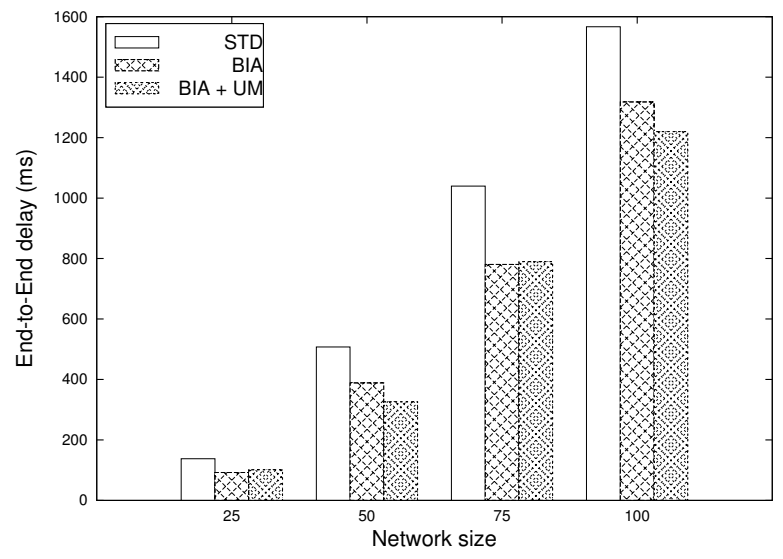

(b) Event-driven messages

Figure 10: Impact of Network size on Average End-to-End delay: Proportion of event-driven messages $=4 \%$, Inter-packets transmission interval $=15 \mathrm{~s}$

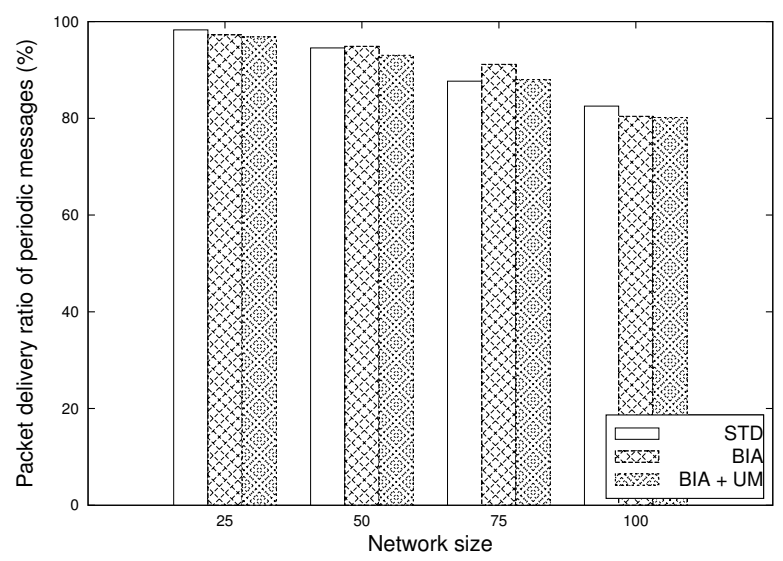

(a) Periodic messages

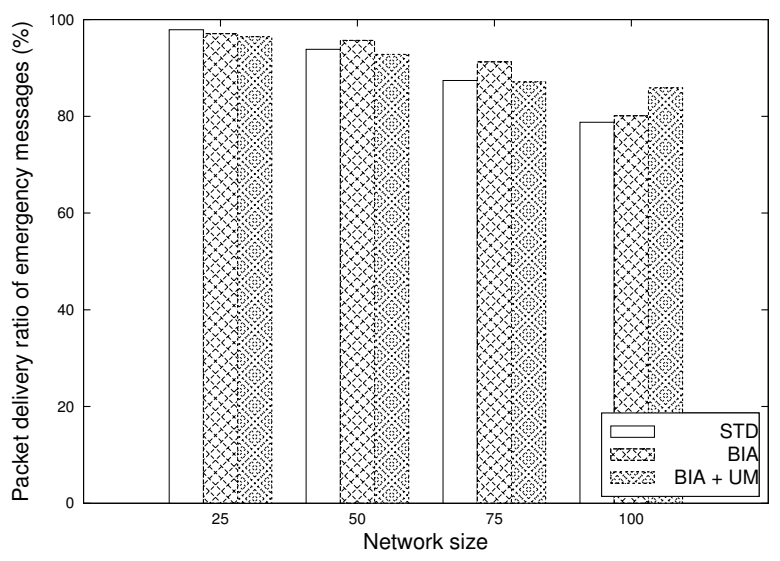

(b) Event-driven messages

Figure 11: Impact of Network size on Packets Delivery Ratio: Proportion of event-driven messages $=4 \%$, Inter-packets transmission interval $=15 \mathrm{~s}$ 